\title{
Exact solution of the Bragg-diffraction problem in sillenites
}

\author{
B. I. Sturman \\ Institute of Automation and Electrometry, Academy of Science, Universitetskiy Prospect 1, Novosibirsk, 630090, Russia \\ D. J. Webb* and R. Kowarschik \\ Institut für Angewandte Optik der Friedrich-Schiller-Universität D-07743 Jena, Germany
}

E. Shamonina and K. H. Ringhofer

Fachbereich Physik der Universität D-49069 Osnabrück, Germany

Received January 3, 1994; revised manuscript received March 25, 1994

\begin{abstract}
A method for the exact solution of the Bragg-diffraction problem for a photorefractive grating in sillenite crystals based on Pauli matrices is proposed. For the two main optical configurations explicit analytical expressions are found for the diffraction efficiency and the polarization of the scattered wave. The exact solution is applied to a detailed analysis of a number of particular cases. For the known limiting cases there is agreement with the published results.
\end{abstract}

\section{INTRODUCTION}

The treatment of light diffraction from a refractive-index grating is one of the elementary problems of optics. For isotropic and optically inactive media its solution is described by the well-known Kogelnik formula. ${ }^{1}$ Various generalizations of this formula are widely used in the nonlinear optics of photorefractive crystals, ${ }^{2}$ in which the spatial variation of the dielectric optical permittivity is linearly related to the photoinduced space-charge field. In particular, the generalization is known for the case of anisotropic diffraction in birefringent crystals. ${ }^{3,4}$ In this case, a simple solution of the problem is possible because the amount of birefringence considerably exceeds the amplitude of the spatial oscillation of the optical dielectric permittivity.

The sillenite crystals (BSO, BTO, BGO) are among the most important and most intensively investigated photorefractive materials. As with any crystals of point group 23 , they are optically isotropic and optically active, and they exhibit a linear electro-optic effect. A number of publications (see, e.g., Refs. 3 and 5-8) were devoted to the theoretical and the experimental investigation of the problem of diffraction from the grating of the spacecharge field. This problem is complex because the amplitude of the spatial variation of the optical dielectric tensor cannot usually be considered to be small in comparison with the contributions to this tensor corresponding to the intrinsic optical activity or the birefringence induced by an external electric field. Under these conditions the description of the intensity and the polarization state of the diffracted wave is reduced to the solution of a set of four differential equations for the complex amplitudes. For the two main optical configurations shown in Fig. 1, this system is well known. ${ }^{5}$ Usually the solution is found either numerically ${ }^{5,6}$ or in the limit of a thin crystal with a small grating amplitude. , $^{7}$

We have succeeded in finding an exact and sufficiently simple solution to the problem of Bragg diffraction from gratings in sillenite crystals for both optical configurations. This solution describes the intensity and the polarization state of the diffracted wave. Below we describe the solution procedure, report its results, analyze the final formulas, and compare them with the known limiting cases.

In this paper we do not touch on the question of the recording of the grating, which should be considered to be an independent process. In particular, it can be performed by separate pump beams without any self-diffraction. Note, however, that the Kukhtarev equations, ${ }^{9}$ which make explicit the dependence of the grating amplitude on the diffusion field, external field, etc., are not sufficient to deal with the problem when enhancement techniques are involved.,10

\section{BASIC EQUATIONS}

Let the space-charge field in the crystal be given by

$$
E_{\mathrm{sc}}=E_{g} \cos \mathbf{K} \cdot \mathbf{r},
$$

where $E_{g}$ is the grating amplitude and $\mathbf{K}=(K, 0,0)$ is the grating vector. This expression is applied to both the transverse $(\mathbf{K} \perp$ [001]) and the longitudinal (K \| [001]) geometries (see Fig. 1). The electric field $\mathcal{E}$ of the light wave can be expressed in the form

$$
\boldsymbol{E}=\left[\mathbf{A}_{1} \exp \left(i \mathbf{k}_{1} \cdot \mathbf{r}\right)+\mathbf{A}_{2} \exp \left(i \mathbf{k}_{2} \cdot \mathbf{r}\right)\right] \exp (-i \omega t)+\text { c.c. },
$$

where $\omega$ is the frequency, $\mathbf{k}_{1,2}$ are the wave vectors, $\mathbf{A}_{1,2}$ are the complex, slowly varying amplitudes with dominating $x$ and $y$ components, and c.c. denotes complex conjugate. The wave vectors have equal length $k_{1,2}=n \omega / c$ ( $n$ is the refractive index) and satisfy the Bragg condition $\mathbf{K}=\mathbf{k}_{1}-\mathbf{k}_{2}$. In agreement with this condition the 


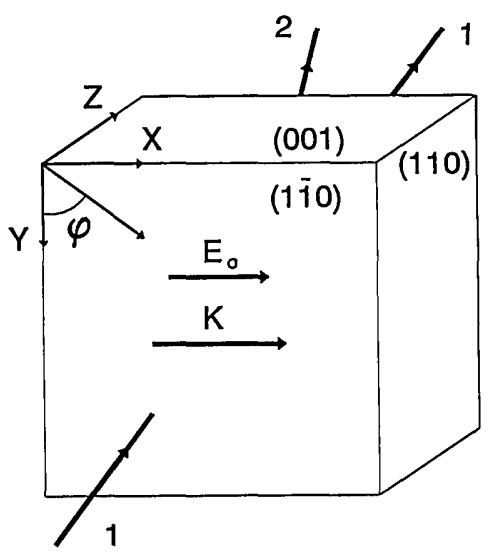

(a)

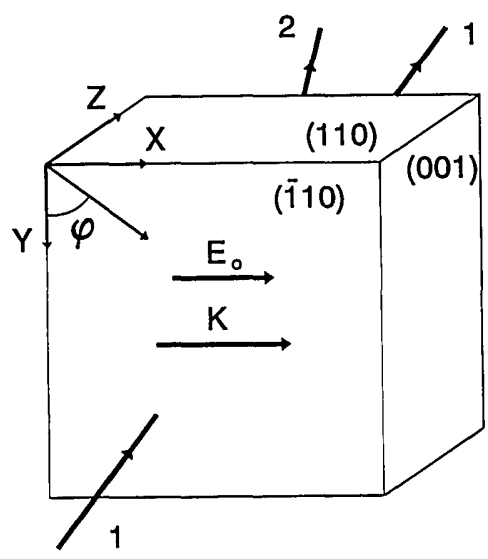

(b)

Fig. 1. (a) Transverse and (b) longitudinal optical configurations.

transverse component of the wave vector of the input wave (let the latter be wave 1 ) is $\mathbf{K} / 2$.

The equations for the amplitudes $\mathbf{A}_{1,2}$ in the case of Bragg diffraction are well known (see, e.g., Ref. 5). We represent these equations in a compact form, using the Pauli matrices, which are the ideal tool when dealing with two-dimensional complex vectors. ${ }^{11,12}$ These matrices have already been used for the investigation of twobeam coupling in sillenites. ${ }^{10,13}$ The Pauli matrices

$$
\hat{\sigma}_{1}=\left[\begin{array}{ll}
0 & 1 \\
1 & 0
\end{array}\right], \quad \hat{\sigma}_{2}=\left[\begin{array}{rr}
0 & -i \\
i & 0
\end{array}\right], \quad \hat{\sigma}_{3}=\left[\begin{array}{rr}
1 & 0 \\
0 & -1
\end{array}\right]
$$

can be considered to be the three components of the matrix-valued $\hat{\boldsymbol{\sigma}}$ vector. These Hermitian matrices have a number of remarkable properties, the most fundamental of which may be expressed in the form

$$
\hat{\sigma}_{j} \cdot \hat{\sigma}_{l}=\delta_{j l} \hat{I}+i \epsilon_{j l m} \hat{\sigma}_{m},
$$

where each of the indices $j, l$, and $m$ takes the values $1 \ldots 3, \hat{I}$ is the $2 \times 2$ unit matrix, $\delta_{j l}$ is the Kronecker symbol, and $\epsilon_{j l m}$ is the Levi-Cività symbol. It follows from Eq. (4) that any function of the $\hat{\sigma}$ matrices can be reduced to a linear one.
In terms of $\hat{\sigma}$ matrices, the equations for the wave amplitudes in the transverse and the longitudinal geometries can be represented in the common form

$$
\begin{aligned}
& \frac{\mathrm{d} \mathbf{A}_{1}}{\mathrm{~d} z}=i \kappa(\mathbf{n} \cdot \hat{\boldsymbol{\sigma}}) \mathbf{A}_{1}+i g \hat{\beta} \mathbf{A}_{2}, \\
& \frac{\mathrm{d} \mathbf{A}_{2}}{\mathrm{~d} z}=i \kappa(\mathbf{n} \cdot \hat{\boldsymbol{\sigma}}) \mathbf{A}_{2}+i g \hat{\beta} \mathbf{A}_{1} .
\end{aligned}
$$

The parameters $\kappa$ and $g$ being introduced into Eqs. (5) as well as the unit vector $\mathbf{n}$ are real quantities. The parameter $\kappa$ is the absolute value of the correction to the $z$ component of the wave vector that is due to optical activity and birefringence induced by the external field. The sign of this correction is opposite for the two optical eigenmodes. The unit vector $\mathbf{n}$ defines the polarization properties of the eigenmodes. The parameter $g$ is the coupling constant describing the strength of coupling of waves 1 and 2 ; the Hermitian matrix $\hat{\beta}$ specifies the polarization property of this coupling.

The value of the coupling constant $g$ does not depend on the type of the optical configuration:

$$
g=\frac{\pi n^{3} r_{41}}{2 \lambda} E_{g},
$$

where $r_{41}$ is the electro-optic coefficient and $\lambda$ is the freespace wavelength. The expressions for $\kappa, \mathbf{n}$, and $\hat{\beta}$ are different for the transverse and the longitudinal geometries:

$$
\perp \text { case: } \kappa=\sqrt{\rho^{2}+s^{2}}, \quad \mathbf{n}=\left(\frac{s}{\kappa},-\frac{\rho}{\kappa}, 0\right),
$$

$$
\begin{gathered}
\hat{\beta}=\hat{\sigma}_{1}, \\
\hat{n} \text { case: } \kappa=\sqrt{\rho^{2}+\frac{s^{2}}{4}}, \quad \mathbf{n}=\left(0,-\frac{\rho}{\kappa}, \frac{s}{2 \kappa}\right), \\
\hat{\beta}=\frac{1}{2}\left(\hat{\sigma}_{3}-\hat{I}\right) .
\end{gathered}
$$

Here $\rho$ is the rotatory power, $s=\pi n^{3} r_{41} E_{0} / \lambda$, and $E_{0}$ is the external electric field. We stress that the set of Eqs. (5) is only a reformulation of the standard equations given in Ref. 5. Technical details can be found in Ref. 13.

The boundary conditions for the wave amplitudes have the form

$$
\mathbf{A}_{1}(0)=\mathbf{A}_{1}{ }^{0}, \quad \mathbf{A}_{2}(0)=0 .
$$

Our aim is to find the diffraction efficiency $\eta=$ $\left|\mathbf{A}_{2}(z) / \mathbf{A}_{1}{ }^{0}\right|^{2}$ and the polarization state of the diffracted wave 2

The linear light absorption is not included in Eqs. (5) because it does not change the polarization characteristics of the diffracted wave and decreases the diffraction efficiency only by a factor $\exp (-\alpha z)$, where $\alpha$ is the absorption coefficient. ${ }^{5}$

As is well known (see, e.g., Refs. 14 and 15), there are several methods for the description of a polarization state: the polarization matrix, the Stokes parameters, representation by a complex number, the Jones vector, and specification by ellipticity and inclination angle. To express the general relations of the theory, the first three methods are most useful. The complex polarization matrix $\hat{P}$ 


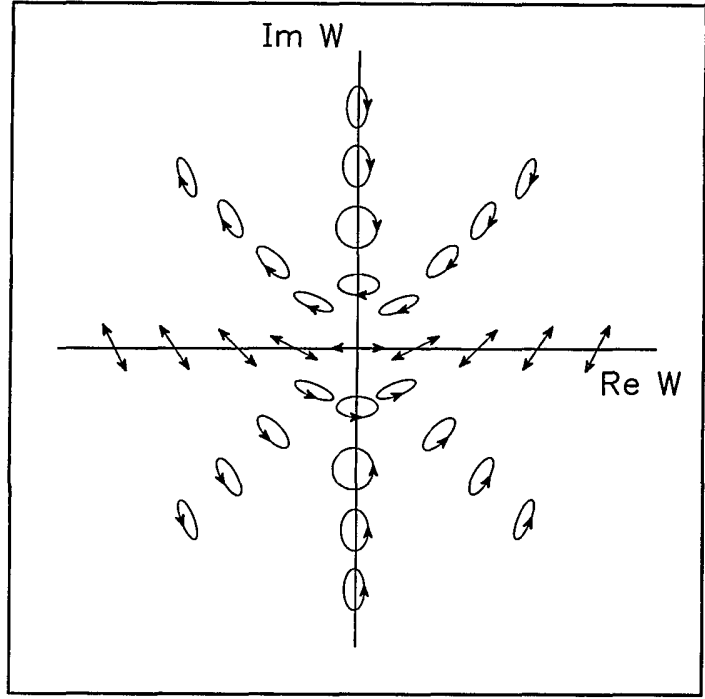

Fig. 2. Complex $W$ representation of a polarization state.

(with matrix elements $P_{\alpha \beta}$ ), the real Stokes parameters $\xi=\left(\xi_{1}, \xi_{2}, \xi_{3}\right)$, and the complex parameter $W$ corresponding to these methods can be expressed through the complex wave amplitude $\mathbf{A}=\left(A_{x}, A_{y}\right)$ as follows:

$$
P_{\alpha \beta}=\frac{A_{\alpha} A_{\beta}{ }^{*}}{|\mathbf{A}|^{2}}, \quad \hat{P}=\frac{1}{2}(\hat{I}+\xi \cdot \hat{\sigma}), \quad W=\frac{A_{y}}{A_{x}} .
$$

The parameters $\xi_{1,3}$ characterize the degree of linear polarization, and the parameter $\xi_{2}$ characterizes the degree of ellipticity. These parameters are expressed by the amplitudes as $\xi_{i}=\operatorname{Tr}\left(\hat{P} \hat{\sigma}_{i}\right)$. We have $\xi_{1}{ }^{2}+\xi_{2}{ }^{2}+\xi_{3}{ }^{2}=1$ for a totally polarized wave. In the special case of linear polarization with an angle $\varphi$ between the polarization vector and the $x$ axis, we have $\xi_{1}=\sin (2 \varphi), \xi_{2}=0, \xi_{3}=\cos (2 \varphi)$, $W=\tan (\varphi)$; for right and left circular polarizations, we have $\xi_{1,3}=0, \xi_{2}= \pm 1, W= \pm i$. A detailed description of the properties of the introduced parameters and the relationships among the different representations can be found in the texts cited in Refs. 14 and 15. We present only one illustration of the efficiency of the $W$ representation of the polarization state: Fig. 2 shows the relation between points of the complex $W$ plane and the polarization properties. ${ }^{15}$

Let the solution of Eqs. (5) have the form

$$
\mathbf{A}_{2}=\hat{T} \mathbf{A}_{1}{ }^{0},
$$

with the evolution matrix $\hat{T}(z)$. Then the diffraction efficiency $\eta$ and the polarization matrix $\hat{P}$ of the diffracted wave 2 may be represented in the general form

$$
\begin{aligned}
& \eta=\operatorname{Tr}\left(\hat{T}^{\dagger} \hat{T} \hat{P}^{0}\right), \\
& \hat{P}=\eta^{-1} \hat{T} \hat{P}^{0} \hat{T}^{\dagger},
\end{aligned}
$$

where $\hat{P}^{0}$ is the polarization matrix of the input wave 1 , and $\hat{T}^{\dagger}$ is the Hermitian conjugate of the matrix $\hat{T}$.

The matrix $\hat{T}$ (and indeed any $2 \times 2$ matrix) can be expressed by $\hat{\sigma}$ matrices:

$$
\hat{T}=a \hat{I}+\mathbf{b} \cdot \hat{\boldsymbol{\sigma}} .
$$

The complex parameters $a(z)$ and $\mathbf{b}(z)$ depend on the optical configuration and on the numbers $\rho, s$, and $g$. Using Eqs. (9), (11), and (12), we can express $\eta$ and $\xi$ by using $a, \mathbf{b}$, and the Stokes parameters of the input wave $\xi_{0}$ :

$$
\begin{aligned}
\eta= & |a|^{2}+|\mathbf{b}|^{2}+\xi_{0}\left(a \mathbf{b}^{*}+a^{*} \mathbf{b}+i \mathbf{b}^{*} \times \mathbf{b}\right), \\
\eta \xi= & i \mathbf{b} \times \mathbf{b}^{*}+\left(|a|^{2}-|\mathbf{b}|^{2}\right) \xi_{0} \\
& +\left[a^{*} \mathbf{b}+i a^{*} \mathbf{b} \times \xi_{0}+\mathbf{b}\left(\mathbf{b}^{*} \cdot \xi_{0}\right)+\text { c.c. }\right] .
\end{aligned}
$$

Instead of using Eq. (13b) to describe the polarization state of the diffracted wave, it is often easier to use the following relationship between the complex parameters $W=A_{2 y} / A_{2 x}$ and $W_{0}=A_{1 y}{ }^{0} / A_{1 x}{ }^{0}$, which follows directly from Eqs. (10) and (12):

$W=\frac{\left(1-d_{z}\right) W_{0}+d_{+}}{d_{-} W_{0}+d_{z}+1}, \quad d_{ \pm}=\frac{b_{x} \pm i b_{y}}{a}, \quad d_{z}=\frac{b_{z}}{a}$.

Therefore $W$ is a fractional-linear function of $W_{0}$, and a study of the change of polarization is reduced to an analysis of this elementary function of a complex argument. Knowing the parameter $d_{z,+,-}$ and the initial polarization state, i.e., a point $W_{0}$ in Fig. 2, one can find the corresponding value of $W$ and, consequently, the polarization of the diffracted beam.

We stress that Eqs. (11), (13), and (14) are merely consequences of conventional physical definitions and mathematical transformations. All the real physical information is contained in the parameters $a$ and $\mathbf{b}$. In Section 3 we find the exact solution of the set of Eqs. (5), and we find these parameters for the transverse and the longitudinal geometries.

\section{EXACT SOLUTIONS}

To solve Eqs. (5) we introduce the new amplitudes $\mathbf{A}_{ \pm}=$ $\mathbf{A}_{1} \pm \mathbf{A}_{2}$. In these variables the coupled system of vectorial equations is split into two separate systems:

$$
\frac{\mathrm{d} \mathbf{A}_{ \pm}}{\mathrm{d} z}=i \hat{G}_{ \pm} \mathbf{A}_{ \pm}, \quad \hat{G}_{ \pm}=\kappa \mathbf{n} \cdot \hat{\boldsymbol{\sigma}} \pm g \hat{\beta} .
$$

It is well known that the solution of an equation such as this is given by an exponential function with the argument $\hat{G}_{ \pm} z \cdot{ }^{11,12}$ Therefore we obtain the solution for the amplitude $\mathbf{A}_{2}$ with the boundary conditions of Eqs. (8) in the form of Eq. (10) with the evolution matrix $\hat{T}$ given by the relation

$$
\hat{T}=(1 / 2)\left[\exp \left(i \hat{G}_{+} z\right)-\exp \left(i \hat{G}_{-} z\right)\right] .
$$

Furthermore, with the help of the Euler formula for matrices,

$$
\exp (i \varphi \mathbf{n} \cdot \hat{\boldsymbol{\sigma}})=\hat{I} \cos (\varphi)+i \mathbf{n} \cdot \hat{\boldsymbol{\sigma}} \sin (\varphi),
$$

which is valid for any $\varphi$ and unit vector $\mathbf{n}$, it is easy to find the explicit form of the parameters $a$ and $\mathbf{b}$ of Eq. (12). For the transverse and the longitudinal geometries, we 
find that

$$
\begin{array}{ll}
\perp \text { case: } & a=(1 / 2)\left[\cos \left(\kappa_{+} z\right)-\cos \left(\kappa_{-} z\right)\right], \\
& \mathbf{b}=(i / 2)\left[\mathbf{n}_{+} \sin \left(\kappa_{+} z\right)-\mathbf{n}_{-} \sin \left(\kappa_{-} z\right)\right],
\end{array}
$$

$$
\| \text { case: } \quad \begin{aligned}
a= & (1 / 2)\left[\exp (-i g z / 2) \cos \left(\kappa_{+} z\right)\right. \\
& \left.-\exp (i g z / 2) \cos \left(\kappa_{-} z\right)\right], \\
\mathbf{b}= & (i / 2)\left[\mathbf{n}_{+} \exp (-i g z / 2) \sin \left(\kappa_{+} z\right)\right. \\
& \left.-\mathbf{n}_{-} \exp (i g z / 2) \sin \left(\kappa_{-} z\right)\right]
\end{aligned}
$$

The real parameters $\kappa_{ \pm}$and the new real unit vectors $\mathbf{n}_{ \pm}$ are also different for the two geometries:

$$
\begin{array}{ll}
\perp \text { case: } & \kappa_{ \pm}=\left[\rho^{2}+(s \pm g)^{2}\right]^{1 / 2}, \\
\mathbf{n}_{ \pm} & =\kappa_{ \pm}{ }^{-1}(s \pm g,-\rho, 0), \\
\| \text { case: } & \kappa_{ \pm}=\left[\rho^{2}+\left(\frac{s \pm g}{2}\right)^{2}\right]^{1 / 2}, \\
\mathbf{n}_{ \pm} & =\kappa_{ \pm}^{-1}\left(0,-\rho, \frac{s \pm g}{2}\right) .
\end{array}
$$

As seen from Eqs. (18), the expressions for the parameters $a$ and $\mathbf{b}$ are simplest for the transverse geometry, where $a$ is real and $\mathbf{b}$ is purely imaginary. Moreover, there are only two spatial frequencies $\kappa_{ \pm}$in this case. For the longitudinal geometry $a$ and $\mathbf{b}$ are complex parameters. Besides, there is a third spatial frequency $g / 2$. Note that the external field $E_{0}$ as well as the grating amplitude $E_{g}$ are introduced into Eqs. (19b) with weight $1 / 2$ in comparison with Eqs. (19a). This is a consequence of the electro-optic properties of crystals with point group 23, to which the sillenites belong. The explicit expressions (18), together with the general formulas (13) and (14), represent the complete solution of the problem of Bragg diffraction in sillenites. Knowing the grating amplitude, the external field, and the proper crystal parameters, one can numerically calculate all the observable properties of the diffracted wave. The importance of the expressions obtained is not restricted, however, to such calculations. These expressions may also be used as the basis of a farreaching analytical analysis of observable properties, for optimization and manipulation of the diffraction parameters, and for the consideration of a number of simple and interesting special cases. Below we give a number of examples of the use of these general analytical expressions. The transverse and the longitudinal geometries are considered separately.

\section{DIFFRACTION PROPERTIES FOR THE TRANSVERSE GEOMETRY}

As we have pointed out, the parameter $a$ is real in this case, and the vector $\mathbf{b}$ is purely imaginary. Therefore the vectors $\mathbf{b} \times \mathbf{b}^{*}$ and $\operatorname{Re}\left(a \mathbf{b}^{*}\right)$ in Eq. (13a) are zero. This result means that the diffraction efficiency does not depend on the polarization state of the input wave. For linear polarization of the input beam, this fact is known from the numerical results of Ref. 5 and also from analytical calculations, restricted to a small grating amplitude and a small crystal thickness. ${ }^{7}$ It is also supported by experimental results. ${ }^{5}$

Using Eqs. (13a) and (18a), we arrive at the following exact expression for the diffraction efficiency:

$$
\begin{aligned}
\eta= & (1 / 2)\left[1-\cos \left(\kappa_{+} z\right) \cos \left(\kappa_{-} z\right)\right. \\
& \left.-\mathbf{n}_{+} \cdot \mathbf{n}_{-} \sin \left(\kappa_{+} z\right) \sin \left(\kappa_{-} z\right)\right] .
\end{aligned}
$$

Let us consider various limiting cases. If we use $\rho=0$ and $E_{0}=0$, Eq. (20) becomes a Kogelnik-like formula, $\eta=\sin ^{2}(g z)$. If only the external field is zero, Eq. (13a) gives

$$
\eta=\frac{g^{2}}{\rho^{2}+g^{2}} \sin ^{2}\left[z \sqrt{\left(\rho^{2}+g^{2}\right)}\right] .
$$

This formula clearly shows the negative role of optical activity. Large values of $\eta$, i.e., $\eta \approx 1$, are possible only for sufficiently large grating amplitudes, $g \gtrless \rho$. In BSO and BGO, $g \approx \rho$ only for unrealistically large amplitudes, $E_{g} \approx(20-25) \mathrm{kV} / \mathrm{cm}$. In BTO this value is approximately three times smaller because of the low rotatory power. This can lead to competition between the rotatory power and the grating amplitude and to oscillation of the diffraction efficiency. Characteristic dependences $\eta\left(E_{g}\right)$ for BSO and BTO crystals are shown in Fig. 3. Curve 2 of Fig. 3(a) gives the impression of saturation, and curve 3 of Fig. 3(b) even appears to indicate decrease; in fact, these curves show the beginning of oscillation.

For the experimentally relevant case of a small grating amplitude, $g \ll \kappa$, it is not difficult to find, from Eqs. (19a) and (20), that

$$
\eta=\sin ^{2}\left(\frac{s g z}{\kappa}\right)+\frac{g^{2} \rho^{2}}{\kappa^{4}} \sin ^{2}(\kappa z) \cos ^{2}\left(\frac{s g z}{\kappa}\right) .
$$

Equation (22) is obtained without any strong restriction on the crystal thickness $z$. It is converted into Eq. (18) of Ref. 7, derived for linearly polarized light, only under the additional condition $s g z \ll \kappa$. Therefore the validity of the final results of Mallick et al. ${ }^{7}$ demands not only the smallness of the grating amplitude (as was claimed in Ref. 7) but also the above-mentioned condition on the crystal thickness.

Figure 4 shows an application of the exact Eq. (20) to the analysis of the behavior of the diffraction efficiency in the region of large grating amplitudes and large crystal thicknesses. One can see that this region corresponds to high values of $\eta$ and to high sensitivity of $\eta$ to the external field.

Next we discuss the polarization properties. With Eqs. (18a) it is not difficult to transform Eqs. (14) to the simple form

$$
\begin{aligned}
& W=\frac{W_{0}-Q^{*}}{W_{0} Q+1}, \\
& Q=\frac{\left(n_{y}{ }^{+}+i n_{x}^{+}\right) \sin \left(\kappa_{+} z\right)-\left(n_{y}{ }^{-}+i n_{x}^{-}\right) \sin \left(\kappa_{-} z\right)}{\cos \left(\kappa_{+} z\right)-\cos \left(\kappa_{-} z\right)} .
\end{aligned}
$$

The polarization properties of the scattered wave are governed by the sole complex parameter $Q$. The zero of the 


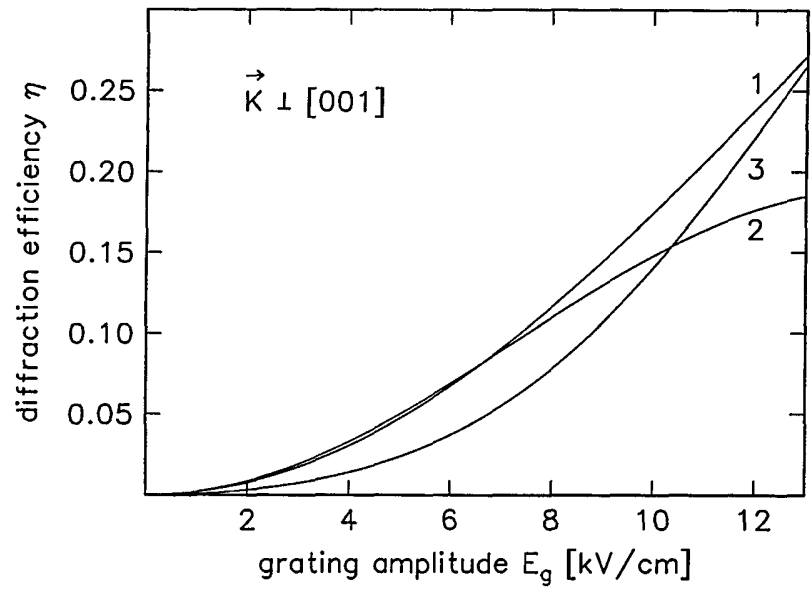

(a)

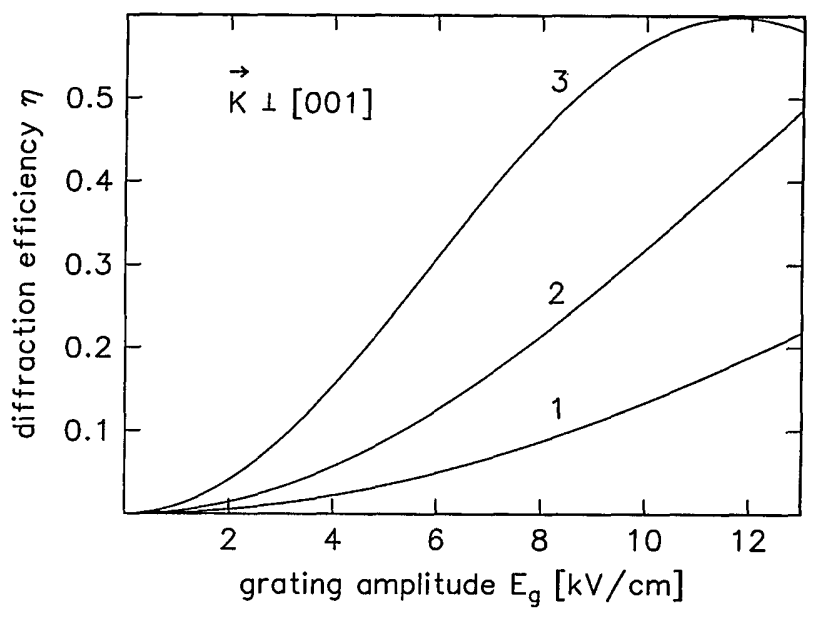

(b)

Fig. 3. Dependence $\eta\left(E_{g}\right)$ in the transverse geometry for (a) BSO $\left(n=2.54, \rho=21.4^{\circ} / \mathrm{mm}, r_{41}=4.5 \mathrm{pm} / \mathrm{V}\right.$ for $\left.\lambda=633 \mathrm{~nm}\right)$ and (b) BTO $\left(n=2.25, \rho=6.3^{\circ} / \mathrm{mm}, r_{41}=4.5 \mathrm{pm} / \mathrm{V}\right.$ for $\lambda=633 \mathrm{~nm}$ ). The external field $E_{0}$ is zero. Curves 1,2 , and 3 correspond to the thicknesses $0.3,0.5$, and $1.0 \mathrm{~cm}$, respectively.

numerator, i.e., the point $W_{0}=Q^{*}$, corresponds to linearhorizontal polarization of the diffracted wave, whereas the zero of the denominator, $W_{0}=-Q^{-1}$, corresponds to vertical polarization. Equations (23) allows one to solve the inverse problem: the calculation of the polarization parameter $W_{0}$ from a given final polarization state $W$. It also permits the possibility of influencing the polarization state, with the dependence of the parameter $Q$ on $E_{0}$.

Let us consider some limiting cases. For the case $E_{0}=$ 0, Eqs. (23) have an especially simple form:

$$
W W_{0}=1
$$

This relation means, in particular, that a linearly polarized input beam with an inclination angle $\varphi_{0}$ becomes a linearly polarized output beam with an angle $\varphi$ given by

$$
\varphi+\varphi_{0}=\pi / 2 .
$$

This feature has already been pointed out in Ref. 7. A circularly left-polarized wave $\left(W_{0}=-i\right)$ becomes a circularly right-polarized wave $(W=i)$, and vice versa. An elliptically polarized input wave changes the direction of rotation, with the new inclination angle being obtained from Eq. (25) (see also Fig. 2).

For small grating amplitudes and a sufficiently thin crystal, $g \ll \kappa$ and $g s z \ll \kappa$, one easily finds that

$$
Q \simeq-\frac{s+i \rho}{\kappa}\left[\frac{\rho}{s \kappa z}+i \cot (\kappa z)\right] .
$$

For $\kappa z \ll 1$ we have $Q \simeq-i / g z \rightarrow i \infty$. In this limit Eqs. (23) again become Eq. (24). Physically the limit $\kappa z \ll 1$ corresponds to extremely thin crystals.

\section{DIFFRACTION PROPERTIES FOR THE LONGITUDINAL GEOMETRY}

The longitudinal geometry is definitely more complicated to deal with analytically than is the transverse geometry. The possibilities of simplifying the general analytical expressions are more restricted here. One can simplify the general result, however, when there is no external field, $E_{0}=0$. This case is quite important because the influence of the external field on the electrooptic properties is much weaker here than for the transverse geometry. Assuming that $E_{0}=0$, we obtain $\kappa_{+}=$ $\kappa_{-}=\tilde{\kappa}=\left(\rho^{2}+g^{2} / 4\right)^{1 / 2}$, and we find that, for the diffraction efficiency,

$$
\begin{aligned}
\eta= & \sin ^{2} \frac{g z}{2}+\frac{g^{2}}{4 \tilde{\kappa}^{2}} \sin ^{2}(\tilde{\kappa} z) \cos (g z) \\
& +\frac{g}{2 \tilde{\kappa}} \sin (\tilde{\kappa} z) \sin (g z)\left[\xi_{1}{ }^{0} \frac{\rho}{\tilde{\kappa}} \sin (\tilde{\kappa} z)-\xi_{3}{ }^{0} \cos (\tilde{\kappa} z)\right] .
\end{aligned}
$$

The expression in brackets describes the dependence of $\eta$ on the polarization state of the input wave. It does not depend explicitly on the Stokes parameter $\xi_{2}{ }^{0}$, which characterizes the degree of ellipticity. For a circularly polarized input wave $\xi_{2}{ }^{0}= \pm 1$ and $\xi_{1}{ }^{0}=\xi_{3}{ }^{0}=0$, with the diffraction efficiency being given here by the first two

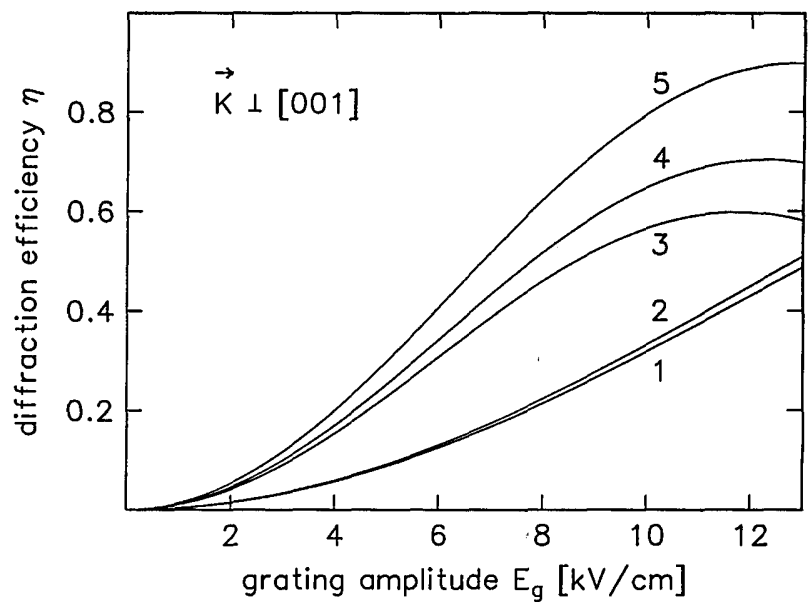

Fig. 4. Dependence $\eta\left(E_{g}\right)$ in the transverse geometry for different values of the external field and the crystal thickness. Curves 1 and 2 correspond to $E_{0}=0$ and $15 \mathrm{kV} / \mathrm{cm}$ with $z=0.5 \mathrm{~cm}$. Curves 3,4 , and 5 correspond to the cases in which $E_{0}$ is 0,5 , and $10 \mathrm{kV} / \mathrm{cm}$, respectively, with $z=1.0 \mathrm{~cm}$. The parameters $n, \rho$, and $r_{41}$ used are typical for BTO [see Fig. 3(b)]. 


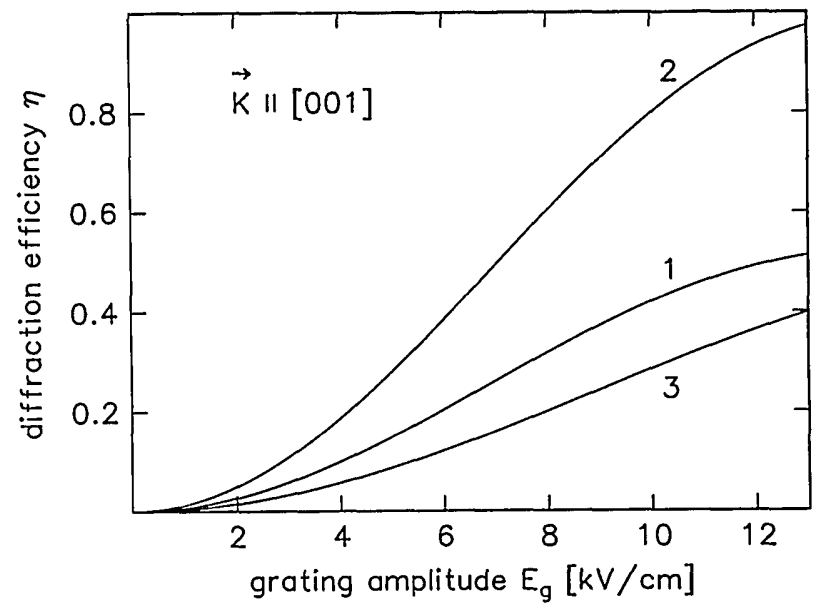

Fig. 5. Dependence $\eta\left(E_{g}\right)$ in the longitudinal geometry for different polarizations of the incident beam, typical parameters of BTO, $z=1.0 \mathrm{~cm}$, and $E_{0}=0$. Curves 1,2 , and 3 correspond to circular polarization $\left(\left|\xi_{2}{ }^{0}\right|=1\right)$, linear polarization with $\varphi_{0}=45^{\circ}\left(\xi_{1}{ }^{0}=1\right)$, and linear horizontal polarization $\left(\xi_{3}{ }^{0}=1\right)$, respectively.

terms in Eq. (27). For a linearly polarized wave $\xi_{1}{ }^{0}=$ $\sin \left(2 \varphi_{0}\right), \xi_{2}{ }^{0}=0$, and $\xi_{3}{ }^{0}=\cos \left(2 \varphi_{0}\right)$, where $\varphi_{0}$ is the inclination angle with respect to the $x$ axis. Hence, using circular and linear polarization, we can extract each of the three main contributions to the diffraction efficiency [the first line on the right-hand side of Eq. (27), the first and the second terms on the second line], which correspond to $\left|\xi_{2}{ }^{0}\right|=1, \xi_{1}{ }^{0}=1$, and $\xi_{3}{ }^{0}=1$. The excess of information derived from experiments with circularly polarized light and with $\varphi_{0}=0, \pi / 4, \pi / 2$, and $3 \pi / 4$ can help to provide additional information. For example, this information can be used as a check on the spatial homogeneity of the permittivity grating or on the fulfillment of the Bragg condition.

In Fig. 5 we show the dependences $\eta\left(E_{g}\right)$ for typical parameters of BTO and for three different polarization states of the input wave. One can see that the diffraction efficiency can approach one for a linearly polarized incident beam with $\varphi_{0}=45^{\circ}$ and for a sufficiently large grating amplitude.

For a weak grating and a moderately thin crystal, $g \ll$ $2 \rho, g z \ll 1$, Eq. (27) simplifies to

$$
\begin{aligned}
\eta= & \frac{g^{2} z^{2}}{4}\left\{1+\frac{\sin ^{2}(\rho z)}{\rho^{2} z^{2}}+\frac{2 \sin (\rho z)}{\rho z}\right. \\
& \left.\times\left[\xi_{1}{ }^{0} \sin (\rho z)-\xi_{3}{ }^{0} \cos (\rho z)\right]\right\} .
\end{aligned}
$$

This formula generalizes Eq. (35) of Ref. 7 to arbitrary polarization of the input beam. It is useful for the analysis of most of the experimental situations for BSO and BGO crystals in the absence of an external field. For a linearly polarized input wave $\xi_{1}{ }^{0} \sin (\rho z)-\xi_{3}{ }^{0} \cos (\rho z)=$ $-\cos \left(\rho z+2 \varphi_{0}\right)$.

Maximum simplification of Eq. (28) is achieved for extremely thin crystals, $\rho z \ll 1, g z \ll 1$; then $\eta \approx g^{2} z^{2}(1-$ $\left.\xi_{3}{ }^{0}\right) / 2$. The diffraction efficiency is maximal here for vertical polarization $\left(\varphi_{0}=0\right)$, zero for horizontal polarization $\left(\varphi_{0}=\pi / 2\right)$, and medium for both circular polarizations of the input beam. This feature is related only to the electro-optical properties of the sillenites.

To investigate the polarization properties of the diffracted wave, it is necessary to calculate the parameters $d_{ \pm}$and $d_{z}$ that are introduced into the general Eq. (14). Using Eqs. (18b), one easily finds that, in the absence of an external field,

$$
\begin{aligned}
d_{ \pm} & = \pm \frac{\rho}{\tilde{\kappa}} \tan (\tilde{\kappa} z) \\
d_{z} & =-\frac{g}{2 \tilde{\kappa}} \tan (\tilde{\kappa} z) \cot (g z / 2) .
\end{aligned}
$$

Note that all these parameters are real. This means, in particular, that a linearly polarized wave diffracts again into a linearly polarized wave with a different inclination angle. The zeros of the numerator and the denominator of Eq. (14) thereby give the values of the inclination angles $\varphi_{0}$, for which an initial liner polarization becomes horizontal and vertical, respectively. The fulfillment of the condition $\tilde{\kappa} z=m \pi(m=1,2, \ldots)$ results in the conservation of the polarization state, whereas the fulfillment of the condition $g z=2 \pi m$ corresponds to a change of the sign of the ellipticity and of the inclination angle for the ellipse (or the vector of linear polarization). In the limit of thin crystals, $\rho z \ll 1, d_{ \pm}$becomes zero, $d_{z}$ becomes -1 , and the ratio $W / W_{0}$ [in agreement with Eq. (14)] becomes infinity. This result means that, for the outgoing wave, the inclination of the polarization vector becomes vertical.

An external field makes the diffraction process more complicated. The diffraction efficiency depends here on the degree of ellipticity of the polarization of the input wave.

\section{DISCUSSION}

Let us summarize and discuss the results obtained. A relatively simple method for the exact solution of the problem of Bragg diffraction for sillenites is proposed. Explicit analytical expressions for the diffraction efficiency and the polarization of the diffracted wave are found for the two principal optical configurations. These exact expressions are applicable to the detailed consideration of a number of important specific situations and features of diffraction. For the known limiting cases, there is agreement of our expressions with the published results.

We expect that the foregoing analysis of special cases does not exhaust the potential of the proposed method and of the general analytic expressions. These expressions may be used for direct numerical calculations as well as for the search for new experimental possibilities.

In the framework of the proposed method, it is not difficult to investigate the polarization properties of the transmitted wave, nor is it difficult to find explicit expressions for the Bragg diffraction of two input beams from a fixed grating. This kind of generalization is well known for the case of isotropic diffraction ${ }^{2}$; it is important for the characterization of photorefractive gratings. Our analytical expressions for $\eta\left(\xi_{0}\right)$ remain valid for the diffraction of a partially polarized input wave.

Note that the generalization of our exact solutions to the case of off-Bragg diffraction is not obvious. 


\section{ACKNOWLEDGMENTS}

We acknowledge the support of B. I. Sturman by the Deutsche Forschungsgemeinschaft within a program supporting the visits of scientists from Russia to Germany and the support of this research within the framework of Sonderforschungsbereich 225. D. J. Webb thanks the Deutscher Akademischer Austauschdienst for supporting his research in Germany.

*Permanent address, Applied Optics Group, Physics Laboratory, The University, Canterbury, Kent, CT2 7NR, United Kingdom.

\section{REFERENCES}

1. H. Kogelnik, "Coupled wave theory for thick hologram gratings," Bell Syst. Tech. J. 48, 2910-2947 (1969).

2. P. Günter and J.-P. Hiugnard, eds., Photorefractive Materials and Their Applications I, Vol. 61 of Topics in Applied Physics (Springer-Verlag, Berlin, 1988), Chap. 2, p. 7.

3. M. P. Petrov, S. I. Stepanov, and A. V. Khomenko, Photorefractive Crystals in Coherent Optical Systems, Springer Series in Optical Sciences (Springer-Verlag, Berlin, 1991), Chap. 5, p. 64.

4. B. I. Sturman and V. M. Fridkin, The Photovoltaic and Photorefractive Effect in Noncentrosymmetric Materials (Gordon \& Breach, New York, 1992), Chap. 3, p. 110.
5. A. Marrakchi, R. V. Johnson, and J. A. R. Tanguay, "Polarization properties of photorefractive diffraction in electrooptic and optically active sillenite crystals (Bragg regime)," J. Opt. Soc. Am. B 3, 321-336 (1986).

6. F. Vachss and L. Hesselink, "Holographic beam coupling in anisotropic photorefractive media," J. Opt. Soc. Am. A 4, 325-339 (1987).

7. S. Mallick, D. Rouède, and A. G. Apostolidis, "Efficiency and polarization characteristics of photorefractive diffraction in a $\mathrm{Bi}_{12} \mathrm{SiO}_{20}$ crystal," J. Opt. Soc. Am. B 4, 1247-1259 (1987).

8. A. Brignon and $K$. H. Wagner, "Polarization state evolution and eigenmode switching in photorefractive BSO," Opt. Commun. 101, 239-246 (1993).

9. N. V. Kukhtarev, V. B. Markov, S. G. Odulov, M. S. Soskin, and V. L. Vinetskii, "Holographic storage in electrooptic crystals," Ferroelectrics 22, 949-964 (1979).

10. D. A. Fish, A. K. Powell, T. J. Hall, P. M. Jeffrey, and R. W. Eason, "Theoretical analysis of mechanism of photorefractive enhancement of photochromic gratings in BSO," Opt. Commun. 98, 349-356 (1993).

11. L. L. Landau and E. M. Lifshitz, Quantum Mechanics (Pergamon, Oxford, 1969), Chap. 8, p. 207.

12. E. Merzbacher, Quantum Mechanics (Wiley, New York, 1970), Chap. 12, p. 271.

13. P. D. Foote, "Optically induced anisotropic light diffraction in photorefractive crystals," Ph.D. dissertation (University of London, London, 1987).

14. L. L. Landau and E. M. Lifshitz, Field Theory (Pergamon, Oxford, 1969), Chap. 6, p. 142.

15. A. Yariv and P. Yeh, Optical Waves in Crystals (Wiley, New York, 1984), Chap. 3, p. 54. 